\title{
EFICIÊNCIA AGRONÔMICA DE COMPOSTOS ORGANOMINERAIS OBTIDOS PELO PROCESSO HUMIFERT $\left({ }^{1}\right)$
}

\author{
DANIELA DA SILVA BENEDITO $\left({ }^{2 *}\right)$; LUÍS IGNÁCIO PROCHNOW $\left({ }^{3}\right)$; ALINE CARNEIRO SILVEROL $\left({ }^{4}\right)$; \\ MARIA CRISTINA MOTTA DE TOLEDO $\left({ }^{5}\right)$
}

\begin{abstract}
RESUMO
A produção tradicional de fertilizantes fosfatados gera grandes volumes de rejeitos e entre estes estão materiais ricos em $\mathrm{P}$, representando um bem mineral inutilizado. Para aproveitar esses rejeitos surgiu o processo Humifert, que tem como objetivo o tratamento ácido da mistura de minérios fosfáticos (elevado ou baixo teor de P) com material orgânico, incrementando a solubilidade do P contido no composto fertilizante, visando aumentar a eficiência agronômica (EA). Este trabalho objetivou avançar a pesquisa sobre o processo Humifert, com a realização de testes de funcionamento do equipamento e experimentos agronômicos, visando avaliar a EA dos compostos obtidos em relação à fonte tradicional de P. Foram realizados dois experimentos agronômicos, sendo um com fosfato monocálcico (FMC), rocha fosfática (RF) de Patos de Minas e turfa, submetidos ou não ao processo Humifert, e foi cultivado milho. O segundo experimento teve como fontes de P o FMC, fosfato reativo de Arad, rejeito de Tapira e turfa, submetidos ou não ao processo Humifert. As culturas utilizadas foram milho e braquiária (três cortes), para avaliar efeito residual das fontes. Em ambos os estudos, avaliou-se a matéria seca da parte aérea (MSPA) das plantas e os dados foram submetidos ao teste de médias. No primeiro experimento, o FMC proporcionou maior MSPA, e a mistura da rocha fosfática Pato de Minas com turfa submetida ao processo foi superior ao controle (sem aplicação de P). No segundo experimento, o FMC foi superior na primeira cultura (milho), e na média dos cultivos Arad foi equivalente ao FMC. Embora tenha havido algumas vantagens em termos de incremento de MSPA devido ao processo, a solubilidade e resultados agronômicos indicam a necessidade de mais pesquisas para adequar as reações e obter melhor qualidade das fontes de P.
\end{abstract}

Palavras-chave: fontes alternativas; fertilizantes fosfatados; matéria orgânica.

$\left.{ }^{1}\right)$ Recebido para publicação em 8 de agosto de 2007 e aceito em 4 de novembro de 2009.

$\left({ }^{2}\right)$ Bolsista CNPq modalidade DTI; Rua Almirante Barroso, 240, ap. 123, 13416-398 Piracicaba (SP). E-mail: danibenedito@gmail.com $\left(^{*}\right)$ Autora correspondente.

$\left({ }^{3}\right)$ Programa IPNI / Brasil; Rua Alfredo Guedes, 1949, Sala 701, 13416-901 Piracicaba (SP). E-mail: lprochnow@ipni.net

$\left({ }^{4}\right)$ Doutoranda do Instituto de Geociências / USP; Rua do Lago, 562, 05508-080 São Paulo (SP). E-mail: alinesilverol@igc.usp.br

$\left(^{5}\right)$ Escola de Artes, Ciências e Humanidades e Pós-Graduação em Geoquímica e Geotectônica, Instituto de Geociências / USP; Rua do Lago, 562, 05508-080 São Paulo (SP). E-mail: mcristol@usp.br 


\title{
ABSTRACT \\ AGRONOMIC EFFECTIVENESS OF ORGANOMINERALS COMPOUNDS OBTAINED BY THE HUMIFERT PROCESS
}

\begin{abstract}
The traditional production process of phosphate fertilizers can generate high amounts of residues with appreciable amounts of $P$ that represent a subutilization of the phosphate rock. With the objective of utilizing these residues the Humifert process was created and consists in the nitric acid treatment of a mixture of phosphatic (low or high contents of $\mathrm{P}$ ) and organic materials in a chamber, with the goal of increasing P solubility and the agronomic effectiveness (AE). The present work has the objectives of generating information relative to the organophosphate compounds from the process. Several tests were applied as related to the operating conditions of the equipment. Two agronomic experiments were conducted. The first experiment tested the Patos de Minas phosphate rock (PR) and organic matter, submitted or not to the Humifert process, with maize as the testing crop. The second experiment used the P sources Arad PR and Tapira residue, and organic matter, also submitted or not to the Humifert process. As to test also the residual effect of the diverse P sources the testing crops were now corn followed by three successive harvests of brachyaria grass. In both experiments the dry-matter yield (DMY) of the crops were evaluated by mean statistics. In the first experiment the MCP lead to higher DMY and the mixture PMT was superior to the control. In the second experiment, although also the MCP was superior in the first crop (maize) in the overall of all harvests the Arad PR was equal to the MCP. Although some advantages in terms of increase in DMY due to the Humifert process, the solubility and agronomic results indicate that more research is necessary as to adequate the reaction to obtain better quality P sources.
\end{abstract}

Key words: alternative sources; phosphate fertilizers; organic matter.

\section{INTRODUÇÃO}

As principais fontes de fósforo (P) utilizadas na agricultura são obtidas industrialmente pelo tratamento de rochas fosfáticas (RF) com ácidos e possuem elevada solubilidade em água, como os superfosfatos e os fosfatos de amônio. Nos solos tropicais agricultáveis, essas fontes têm rápida dissolução, favorecendo a interação do elemento com o solo e reduzindo a disponibilidade de $\mathrm{P}$ às plantas. Nos últimos anos, a utilização de fontes alternativas, como as RF em estado natural (fosfato natural), aumentou acentuadamente, embora esses produtos tenham baixa solubilidade em água quando comparados aos fosfatos solúveis (Horowitz e Meurer, 2003).

A produção de fertilizantes fosfatados gera muitos rejeitos e entre esses estão materiais ainda relativamente ricos em $P$, cujo aproveitamento não é possível pelos atuais métodos industriais, representando, portanto, um bem mineral não utilizado e um problema ambiental preocupante, devido aos grandes volumes envolvidos.

$\mathrm{Na}$ tentativa de utilização desses rejeitos surgiu o processo Humifert, proposto pelos pesquisadores franceses Sternicha e Benard, em 1988 (STERNicha, 1988). O processo objetiva a elaboração de um fertilizante organofosfatado através de técnicas simples, a partir de rocha fosfática (baixo ou alto teor de $\mathrm{P}_{2} \mathrm{O}_{5}$ ) e materiais orgânicos. Consiste em tratar, em reator sob ligeira pressão, a mistura úmida de matéria orgânica triturada, de origem vegetal ou animal, e rochas fosfáticas moídas.
Na presença da umidade da mistura e com a formação de óxidos de nitrogênio na coluna do reator Humifert, ocorre a produção de ácido nítrico nascente. É relatado por STERNICHA (1988) que, com a formação do ácido nítrico na superfície da mistura, a dissolução do material fosfático é favorecida, aumentando a solubilidade e, consequentemente, a eficiência agronômica. A forma físico-química desses compostos pode ser interessante por ocorrer envolvimento das partículas de fosfatos pelos compostos orgânicos, permitindo ao $\mathrm{P}$ ser disponível por um período relativamente mais prolongado, evitando-se a rápida retrogradação. Este contexto se encaixa nas ideias mais modernas de desenvolvimento sustentado, conservação de recursos naturais e tecnologia limpa.

É comentado nos trabalhos dе Ова (2000) е Silverol et al. (2007), e preconizado por Sternicha (1988), que esse processo estudado oferece uma alternativa ao aproveitamento de materiais rejeitados no processo convencional, como minérios inadequados às condições usuais da usina de beneficiamento; rejeitos com teores baixos de $\mathrm{P}_{2} \mathrm{O}_{5}$ (estéreis das frentes de lavra e rejeitos das usinas de beneficiamento); minérios complexos ou inviáveis à obtenção de concentrados que atendam às especificações de mercado; e depósitos pequenos, ricos ou pobres em $\mathrm{P}_{2} \mathrm{O}_{5}$, que não interessam às grandes mineradoras, mas capazes de suprir localizadamente a demanda por fertilizantes fosfatados.

Entretanto, considera-se que estudos no sentido de avaliar a eficiência agronômica dos compostos fertilizantes obtidos a partir do processo 
Humifert ainda são escassos. Há, sem dúvida, necessidade de mais pesquisas que possibilitem, quando analisadas em conjunto, conclusões mais seguras sobre o método proposto. Assim, com este trabalho o objetivo geral foi contribuir com informações relativas à produção e eficiência agronômica dos compostos fertilizantes organominerais produzidos em relação à fonte de elevada solubilidade em água, tradicionalmente empregada. Os objetivos específicos foram: (a) Testes de produção de novos compostos, com materiais não utilizados anteriormente; (b) Aplicação do processo em condições brasileiras; (c) Caracterização (testes de solubilidade de P) das matérias-primas e produtos obtidos; e (d) Avaliação da eficiência agronômica em condições de casa de vegetação.

\section{MATERIAL E MÉTODOS}

Como fontes de fósforo para produção dos compostos organominerais foram utilizados materiais de importância em termos de localização ou de riqueza em reservas totais ou teores, mas contendo fatores limitantes para o aproveitamento tradicional do material enriquecido em $\mathrm{P}_{2} \mathrm{O}_{5}$, e material rejeitado proveniente de usina de mineração. As amostras utilizadas foram provenientes do minério concentrado, de Patos de Minas (MG), coletadas nos poços de pesquisa, e do minério de Tapira (MG), rejeitado no processo tradicional de fabricação de fertilizantes fosfatados. Para efeito de comparação da eficiência agronômica foi utilizado o fosfato natural reativo de Arad. A caracterização dos materiais (Tabela 1) seguiu metodologia de BrAsIL (2004).

Como fonte de matéria orgânica foram utilizadas amostras de turfa (Solomax), compostas por $488 \mathrm{~g} \mathrm{~kg}^{-1}$ de matéria orgânica total, sendo $72,8 \%$ compostável, $197 \mathrm{~g} \mathrm{~kg}^{-1}$ de carbono orgânico e somente $0,6 \mathrm{~g} \mathrm{~kg}^{-1}$ de $\mathrm{P}_{2} \mathrm{O}_{5}$ total. É relatado que há estímulo na absorção de $\mathrm{N}$ e $\mathrm{P}$ na presença de compostos de baixa massa molecular presentes nas substâncias húmicas (NARDI et al., 2005). Por este motivo, a turfa foi escolhida como material orgânico e por ter sido usada em trabalho anterior realizado por Ова (2000), permitindo comparação dos resultados.

Tabela 1. Teor total de $\mathrm{P}_{2} \mathrm{O}_{5}$ e solubilidade dos materiais fosfáticos em citrato neutro de amônio (CNA), ácido cítrico a $2 \%(\mathrm{AC})$ e em água

\begin{tabular}{lcccc}
\hline Materiais fosfáticos & $\mathrm{P}_{2} \mathrm{O}_{5}$ Total & \multicolumn{2}{c}{$\mathrm{P}_{2} \mathrm{O}_{5}$ solúvel } \\
\cline { 3 - 5 } & & $\mathrm{CNA}$ & $\mathrm{AC}$ & \\
\cline { 3 - 5 } & 249 & 11 & 59 & 0 \\
Patos de Minas & 320 & 54 & 114 & 1 \\
Arad & 24 & 2 & 5 & 0 \\
Tapira rejeito &
\end{tabular}

O equipamento Humifert utilizado (ToLEDo et al., 2006; SILVEROL, 2006), montado de acordo com o projeto original cedido por pesquisadores do Instituto Nacional Politécnico de Toulouse (INPT-França), está instalado no Instituto de Geociências da Universidade de São Paulo - USP.

As etapas químicas determinantes do processo são a combustão da amônia, que resulta na formação de óxidos nitrosos; a transformação oxidativa em óxidos nítricos e o ataque dos fosfatos com a formação do ácido nítrico nascente na mistura (BIDEgain, 1995). O equipamento é composto basicamente por três partes, o forno, o recipiente oxidante e o reator. Opera através da passagem de fluxo de ar com óxidos de nitrogênio pela mistura de rocha fosfática com material orgânico úmido (umidade entre $20 \%$ e $30 \%$, dependendo da textura do material utilizado, de modo que permita a passagem dos gases pela mistura) (ROUQUET, 1989).
Foram feitos testes do material no equipamento, com o objetivo de verificar a variação da solubilidade do $\mathrm{P}$ por meio de diferentes extratores (CNA + água, ácido cítrico e água), em função do tempo de exposição ao processo. Em trabalho realizado por SILVEROL (2006), foi definido o tempo de 12 horas para a mistura de rocha fosfática de Angico dos Dias (fonte de P) e lodo de esgoto (material orgânico).

Neste trabalho, foram testados maiores períodos de exposição dos materiais às reações no equipamento Humifert, com o objetivo de verificar se haveria incremento na solubilidade. Foram avaliados os períodos de 12, 16 e 35 horas de exposição da mistura do material de Patos de Minas concentrado (PM) com turfa (T) (PMT), 16 e 35 horas para a mistura do fosfato reativo de Arad (A) com turfa (AT) e 16 horas para a mistura do rejeito de Tapira (Tap) com turfa (TapT). 
As misturas não foram expostas às reações pelo mesmo período de tempo por necessidade de constantes reparos no equipamento nesta fase da pesquisa.

A caracterização dos materiais fosfatados também foi feita de acordo com metodologia de BRASIL (2004), sendo realizada a determinação da quantidade de fósforo extraída através da gravimetria.

A experimentação agronômica foi realizada em casa de vegetação, utilizando vasos com $3 \mathrm{~kg}$ de terra. Para definição da dose a ser utilizada nos experimentos de avaliação da eficiência agronômica dos compostos obtidos pelo processo Humifert, foi realizado um experimento preliminar (curva de resposta), com amostras de terra passadas em peneira de $2 \mathrm{~mm}$ provenientes de Latossolo Vermelho-Amarelo (LVA), coletadas na camada de 0 a $20 \mathrm{~cm}$ de profundidade, na Serra de Itaqueri, no município de São Pedro (SP), com baixo teor de fósforo. A caracterização química, seguindo método de RAIJ et al. (2001), revelou valores de $\mathrm{pH}$ em $\mathrm{CaCl}_{2}$ de 4,0; teor de matéria orgânica de $16 \mathrm{~g} \mathrm{dm}^{-3}$; teores de fósforo e enxofre de $1 \mathrm{e} 6 \mathrm{mg} \mathrm{dm}^{-3}$ respectivamente; teores de potássio, cálcio, magnésio e alumínio de $0,5,1,1$ e 8 $\mathrm{mmol}_{\mathrm{c}} \mathrm{dm}^{-3}$ respectivamente; saturação por bases de $8 \%$ e saturação por alumínio de $76 \%$.

A caracterização granulométrica foi realizada pelo método do densímetro, seguindo método proposto pela EMBrapa (1997), sendo os resultados: 680,20 e $300 \mathrm{~g} \mathrm{~kg}^{-1}$ respectivamente, de areia, silte e argila classificada, de acordo com EMBRAPA (1999), como textura média.

A calagem foi feita para elevar a saturação por bases para 60\% (RAIJ e QuAgGio, 1983) e as amostras de terra foram incubadas com calcário $($ PRNT $=91,8 \%)$ por aproximadamente 15 dias, período em que foi mantida a umidade da terra em $60 \%$ da capacidade de campo. Foi utilizado o delineamento de blocos completos ao acaso com três repetições e cinco doses de P: $0 ; 12,5 ; 25 ; 50$ e 100 $\mathrm{mg} \mathrm{kg}{ }^{-1} \mathrm{P}$, como fosfato monocálcico. A adubação básica consistiu de $200 \mathrm{mg} \mathrm{kg}^{-1} \mathrm{~N}$ (uréia) e $200 \mathrm{mg}$ $\mathrm{kg}^{-1} \mathrm{~K}(\mathrm{KCl})$. Foi adicionada uma solução contendo $5,10,10,10,5$ e $1 \mathrm{mg} \mathrm{kg}^{-1}$ de B, Fe, Mn, Zn, Cu e Mo, sendo o B na forma de ácido bórico, o Mo na forma de molibdato de amônio e os demais na forma de sulfato.

Foram semeadas seis sementes de milho por vaso e as plântulas desbastadas para duas por vaso, aproximadamente dez dias após a emergência (DAE). A terra nos vasos foi umedecida com água destilada, a fim de manter $60 \%$ da capacidade de campo durante o período do experimento.
As plantas foram colhidas aproximadamente $40 \mathrm{DAE}$, secas em estufa a $60{ }^{\circ} \mathrm{C}$ e pesadas, para obtenção da matéria seca produzida pela parte aérea. De acordo com os resultados definiu-se a dose de 75 $\mathrm{mg} \mathrm{kg}^{-1}$ de $\mathrm{P}$ total para utilização nos experimentos com os compostos produzidos pelo equipamento Humifert.

\section{Experimento com rocha fosfática de Patos de Minas e turfa}

Este experimento envolveu nove tratamentos, com quatro repetições, em blocos casualizados, mais um controle, sem aplicação de P. Como fontes de fósforo foram utilizados o fosfato monocálcico p.a. (FMC - fonte padrão de P) e a RF de Patos de Minas (PM) e como material orgânico foi utilizada a turfa (T), submetidos $(\mathrm{PH})$ ou não ao processo Humifert, por 16 horas (Tabela 2).

Tabela 2. Tratamentos do experimento com a rocha fosfática de Patos de Minas (PM) e turfa (T)

\begin{tabular}{ll}
\hline Tratamento & Identificação \\
\hline Controle & sem aplicação de fósforo \\
FMC & fosfato monocálcico \\
PM & PM sem processo ${ }^{(1)}$ \\
PMPH & PM com processo ${ }^{(2)}$ \\
T & turfa sem processo \\
TPH & turfa com processo \\
PMPH+T & PM com processo + turfa sem processo \\
PM+TPH & PM sem processo + turfa com processo \\
PMT & PM sem processo + turfa sem processo \\
PMTPH & PM e turfa passados juntos pelo processo $\left({ }^{3}\right)$ \\
\hline
\end{tabular}

$\left({ }^{1}\right)$ Sem exposição ao equipamento Humifert. $\left({ }^{2}\right)$ Com exposição ao equipamento Humifert.

$\left.{ }^{3}\right)$ Composto de interesse - mistura de material fosfático e orgânico submetidos ao equipamento.

A calagem foi realizada individualmente nos vasos, com o objetivo de elevar a saturação por bases das amostras de solo para $60 \%$. As amostras de solo foram incubadas por aproximadamente 15 dias, período em que foi mantida a umidade em cerca de $60 \%$ da capacidade de campo.

As quantidades de material fosfático aplicadas nos tratamentos, passados ou não pelo processo, foram estipuladas fornecer a dose de $\mathrm{P}$ definida no experimento de curva de resposta, ou seja, $75 \mathrm{mg} \mathrm{kg}^{-1}$ de $\mathrm{P}$ total da mistura ou RF aplicada isoladamente. A quantidade de material orgânico, quando aplicado isoladamente, foi definida em função da quantidade desse material fornecida na mistura Humifert para suprir a dose 
de P estipulada. Os demais procedimentos foram os mesmos daqueles utilizados no experimento de curva de resposta.

O experimento foi realizado no período de 25 / $8 / 2005$ a $27 / 10 / 2005$, data em que as plantas foram colhidas e secas em estufa a $60^{\circ} \mathrm{C}$ até atingirem massa constante, para obtenção da produção de matéria seca. A eficiência agronômica (EAR) da rocha fosfática de Patos de Minas, em relação à fonte de elevada solubilidade em água (FMC) foi obtida a partir da seguinte equação:

$$
\mathrm{EAR}_{\mathrm{i}}=\frac{\mathrm{Y}_{\mathrm{i}}}{\mathrm{Y}_{\mathrm{FMC}}} * 100
$$

sendo: $Y_{i}=$ produção de matéria seca pela fonte $i, e$ $\mathrm{Y}_{\mathrm{FMC}}=$ produção de matéria seca pela fonte padrão, o FMC.

\section{Experimento com fosfato reativo de Arad, rejeito de Tapira e turfa}

Com este experimento, o objetivo foi avaliar a eficiência agronômica das rochas fosfáticas de Arad e rejeito de Tapira comparando ao fosfato monocálcico, submetidos ou não ao processo Humifert, na presença ou ausência da turfa, na dose de $\mathrm{P}$ estabelecida, além de avaliar o efeito residual das fontes.

As recomendações de calagem e adubação básica e a aplicação dos tratamentos obedeceram ao ensaio anterior. As adubações básicas foram realizadas anteriormente à semeadura do milho, da braquiária e a cada corte da braquiária para cultivo subsequente.

Os tratamentos envolveram três fontes de $\mathrm{P}$ (FMC, utilizado como fonte padrão de $\mathrm{P}$, rocha fosfática de Arad - A, e material rejeitado da mina de Tapira - Tap), turfa como material orgânico (T), presença $(\mathrm{PH})$ ou ausência do processo Humifert, uma dose de P (75 $\mathrm{mg} \mathrm{kg}^{-1}$ de $\mathrm{P}$, definida no experimento de curva de resposta), calculada em função do teor total de P da mistura ou RF aplicado isoladamente, e controle (sem aplicação de P)(Tabela 3). A quantidade de material orgânico aplicada foi definida em função da quantidade desse material fornecida na mistura Humifert para suprir a dose de $P$ estipulada. As fontes de $\mathrm{P}$ foram aplicadas somente na época da instalação do experimento, em mistura total com a terra contida nos vasos, antes da semeadura do milho (primeira cultura), a fim de permitir a avaliação do efeito residual das fontes com a cultura da braquiária (três cortes). O delineamento experimental foi de blocos ao acaso com três repetições.
Tabela 3. Tratamentos do experimento com a rocha fosfática de Arad (A), rejeito de Tapira (Tap) e turfa (T)

\begin{tabular}{ll}
\hline Tratamento & Identificação \\
\hline Controle & sem aplicação de fósforo \\
FMC & fosfato monocálcico \\
FMC + T & FMC sem processo + turfa sem processo \\
FMC + TPH & FMC sem processo + turfa com processo \\
A & Arad sem processo $\left({ }^{1}\right)$ \\
APH & Arad com processo $\left({ }^{2}\right)$ \\
A + T & Arad sem processo + turfa sem processo \\
A + TPH & Arad sem processo + turfa com processo \\
APH + T & Arad com processo + turfa sem processo \\
APH + TPH & Arad com processo + turfa com processo $\left({ }^{3}\right)$ \\
ATPH & Arad e turfa passados juntos pelo processo $\left(^{4}\right)$ \\
T & turfa sem processo \\
TPH & turfa com processo \\
Tap & Tapira sem processo \\
TapPH & Tapira com processo \\
Tap + T & Tapira sem processo + turfa sem processo \\
Tap + TPH & Tapira sem processo + turfa com processo \\
TapPH + T & Tapira com processo + turfa sem processo \\
TapPH + TPH & Tapira com processo + turfa com processo $\left({ }^{3}\right)$ \\
TapTPH & Tapira e turfa passados juntos pelo processo $\left({ }^{4}\right)$ \\
\hline
\end{tabular}

$\left({ }^{1}\right)$ Sem exposição ao equipamento Humifert. $\left({ }^{2}\right)$ Com exposição ao equipamento Humifert. $\left({ }^{3}\right)$ Mistura manual dos materiais. $\left({ }^{4}\right)$ Composto de interesse - mistura de material fosfático e orgânico submetidos ao equipamento.

O experimento foi realizado no período de 10/ $4 / 2006$ a 4/2/2007, com quatro cultivos sucessivos, sendo o primeiro com milho (duas plantas por vaso) e os três demais com braquiária (seis plantas por vaso). O primeiro cultivo foi realizado de $9 / 5$ a $18 /$ $6 / 2006$, data em que foram colhidas as plantas de milho. O primeiro cultivo da braquiária foi durante o período $11 / 8$ a $10 / 11 / 2006$, quando ocorreu o primeiro corte. O segundo cultivo da braquiária foi de $11 / 11$ a 20/12/2006 (segundo corte), e de 21/12/ 2006 a 4/2/2007, o terceiro cultivo (terceiro corte). Os demais procedimentos, inclusive o cálculo da EAR, foram os mesmos do experimento A.

\section{RESULTADOS E DISCUSSÃO}

Através dos resultados da tabela 4 pode-se observar que houve incremento na solubilidade dos materiais com a exposição ao equipamento.Observouse aumento de solubilidade em ácido cítrico de $2,4 \%$ (1,7 para $4,1 \%)$ na mistura de Patos de Minas com turfa, quando foi submetida às reações por 12 horas em relação à mistura in natura. Para a mistura do fosfato reativo de Arad com turfa, o aumento foi de 3,4\% (2,7 para $6,1 \%$ ) depois de submetida por 16 horas ao reator. 
Tabela 4. Solubilidade dos materiais em diversos extratores em função do período de exposição ao equipamento Humifert

\begin{tabular}{|c|c|c|c|c|c|}
\hline \multirow{3}{*}{ Materiais } & \multirow[b]{3}{*}{ Total } & \multicolumn{4}{|c|}{$\mathrm{P}_{2} \mathrm{O}_{5}\left(\mathrm{~g} \mathrm{~kg}^{-1}\right)$} \\
\hline & & \multicolumn{4}{|c|}{ Solúvel em } \\
\hline & & $\mathrm{AC}$ & CNA + água & CNA + água / Total & Água \\
\hline \multicolumn{6}{|c|}{$\%$} \\
\hline PMT 0 & 71 & 17 & 3 & 4,2 & 0 \\
\hline PMT 12 & 97 & 41 & 28 & 28,9 & 17 \\
\hline PMT 16 & 98 & 48 & 37 & 37,8 & 20 \\
\hline PMT 35 & 68 & 26 & 13 & 19,1 & 3 \\
\hline AT 0 & 75 & 27 & 13 & 17,3 & 0 \\
\hline AT 16 & 84 & 61 & 24 & 28,6 & 0 \\
\hline AT 35 & 79 & 62 & 25 & 31,6 & 1 \\
\hline TapT 16 & 18 & 4 & 2 & 11,1 & 0 \\
\hline
\end{tabular}

PMT = Patos de Minas + Turfa submetidos juntos ao equipamento; AT = fosfato reativo de Arad + Turfa submetidos juntos ao equipamento; TapT $=$ rejeito de Tapira + Turfa submetidos juntos ao equipamento; 0 = sem exposição ao equipamento Humifert e 12 , 16 e 35 são referentes às horas de exposição ao equipamento Humifert.

Entretanto, esses incrementos em solubilidade foram inferiores aos esperados e considerados pequenos, devendo-se, provavelmente, à criação de canais preferenciais de passagem dos gases pela mistura, acarretando em baixa eficiência de solubilização no reator. Outra explicação para esse fato pode ser a rápida passagem dos gases pela mistura dentro do reator, sendo imediatamente liberados para recipientes reservados para neutralização, impossibilitando maior tempo de contato e, consequentemente, reação dos componentes da mistura com o ácido formado na superfície da mistura.

Verifica-se para a mistura PMT que, variando o período de exposição ao equipamento de 12 para 16 horas houve tendência de aumento de solubilidade. Entretanto, é possível verificar que o aumento de 16 para 35 horas proporcionou queda de solubilidade. Uma possibilidade para explicar este fato seria a formação de novos compostos fosfatados de baixa solubilidade, o que é, a princípio, indesejável no processo. A presença de dados na literatura confirma que certos compostos insolúveis contendo P podem ser eficientes sob o ponto de vista agronômico (Prochnow et al., 2003).

Para a mistura AT, nota-se que a solubilidade do material praticamente não foi alterada quando o tempo de exposição foi aumentado de 16 para 35 horas. Para a mistura do material rejeitado de Tapira com turfa, não foram realizadas comparações devido ao baixo teor de fósforo desse material. Dessa maneira, foi definido que o tempo de exposição dos materiais utilizados nos ensaios agronômicos seria de 16 horas.
Com relação aos experimentos agronômicos, observa-se pelos dados da tabela 5 que houve resposta à adubação fosfatada e que a fonte de $\mathrm{P}$ de elevada solubilidade em água (FMC) proporcionou maior produção de matéria seca pelas plantas de milho. A resposta das plantas ao composto Humifert, ou seja, ao material fosfático de Patos de Minas e à turfa submetidos juntos ao processo (PMTPH) foi intermediária, superior ao controle, porém bastante inferior à fonte de elevada solubilidade $(24 \% \mathrm{em}$ relação ao fosfato monocálcico).

Tabela 5. Produção de matéria seca da parte aérea (MSPA) de plantas de milho e eficiência agronômica relativa das fontes de $\mathrm{P}$

\begin{tabular}{lcc}
\hline Tratamentos & MSPA & EAR \\
\hline Controle & $\mathrm{g}$ & $\%$ \\
FMC & $2,0 \mathrm{c}$ & 12 \\
PM & $16,6 \mathrm{a}$ & 100 \\
PMPH & $2,5 \mathrm{bc}$ & 15 \\
$\mathrm{~T}$ & $2,1 \mathrm{c}$ & 13 \\
TPH & $2,0 \mathrm{c}$ & 12 \\
PMPH + T & $2,1 \mathrm{c}$ & 13 \\
PM + TPH & $2,1 \mathrm{c}$ & 12 \\
PMT & $2,0 \mathrm{c}$ & 12 \\
PMTPH & $1,9 \mathrm{c}$ & 11 \\
\hline $\mathrm{CV}(\%)$ & $3,9 \mathrm{~b}$ & 24 \\
\hline
\end{tabular}

Valores seguidos pela mesma letra não diferem estatisticamente entre si pelo teste de Tukey 5\% de probabilidade. 
Nota-se ter havido diferença significativa entre a produção de MSPA proporcionada pelo composto de interesse (PMTPH) e pela mesma mistura, porém sem passar pelo processo Humifert (PMT), com vantagem para o primeiro, comprovando, portanto, que houve efeito da exposição da mistura ao equipamento Humifert, mesmo que este ainda não tenha sido satisfatório.

Vale também observar que, apesar da produção de MSPA ter sido maior no tratamento PMTPH $(3,9 \mathrm{~g})$, esta não diferiu estatisticamente daquela quando foi adicionada a rocha fosfática de Patos de Minas sem exposição ao processo $(2,5 \mathrm{~g})$, não representando incrementos adicionais em relação a esta fonte.

Esses resultados estão de acordo com a baixa solubilidade dos compostos, indicando o processo pouco eficiente em incrementar a eficiência agronômica do composto, proporcionando produção insatisfatória da cultura e levando à necessidade de mais estudos e, provavelmente, ajuste do equipamento, a aumentar a eficiência das reações.

No segundo experimento, os dados apresentados na tabela 6 permitem avaliar que houve resposta às fontes de $\mathrm{P}$, e que no cultivo do milho a fonte de maior solubilidade (FMC) proporcionou maior produção de MSPA, conforme o experimento anterior. A produção de MSPA das plantas de milho seguiu a ordem geral: fonte de elevada solubilidade (FMC) $>$ rocha fosfática de elevada reatividade (Arad) > material de baixa reatividade $($ Tap $)=$ material orgânico (turfa) $=$ controle $($ sem $\mathrm{P})$. Dentro dos dois grupos (FMC e Arad) não houve diferença entre formas de tratamento, sendo a produção de matéria seca definida basicamente pela fonte de P. Por exemplo, para Arad não houve importância do tipo de tratamento quanto ao processo Humifert, ou mesmo da presença ou não de material orgânico.

Tabela 6. Produção de matéria seca da parte aérea (MSPA) de plantas de milho e braquiária e eficiência agronômica relativa (EAR)

\begin{tabular}{|c|c|c|c|c|c|c|c|c|c|c|}
\hline \multirow[t]{3}{*}{ Tratamentos } & \multirow{2}{*}{\multicolumn{2}{|c|}{ Milho }} & \multicolumn{6}{|c|}{$\begin{array}{c}\text { Braquiária } \\
\text { (efeito residual) }\end{array}$} & \multirow{2}{*}{\multicolumn{2}{|c|}{$\begin{array}{c}\text { Soma } 3 \text { cultivos } \\
\text { de braquiária }\end{array}$}} \\
\hline & & & \multicolumn{2}{|c|}{$1 .^{\circ}$ cultivo } & \multicolumn{2}{|c|}{ 2. ${ }^{\circ}$ cultivo } & \multicolumn{2}{|c|}{$3 .^{\circ}$ cultivo } & & \\
\hline & MSPA & EAR & MSPA & EAR & MSPA & EAR & MSPA & EAR & MSPA & EAR \\
\hline & $\mathrm{g}$ & $\%$ & $\mathrm{~g}$ & $\%$ & $\mathrm{~g}$ & $\%$ & $\mathrm{~g}$ & $\%$ & g & $\%$ \\
\hline Controle & $1,7 \mathrm{c}$ & 14 & $0,4 \mathrm{c}$ & 2 & - & - & - & - & $0,4 \mathrm{c}$ & 1 \\
\hline FMC SH & $12,3 \mathrm{a}$ & 100 & $23,0 \mathrm{a}$ & 100 & $16,5 \mathrm{ab}$ & 100 & $22,5 \mathrm{a}$ & 100 & $62,0 \mathrm{ab}$ & 100 \\
\hline $\mathrm{FMC} \mathrm{SH}+\mathrm{TSH}$ & $11,6 \mathrm{a}$ & 95 & $19,9 \mathrm{ab}$ & 87 & $17,3 \mathrm{ab}$ & 105 & $22,7 \mathrm{a}$ & 101 & $59,9 \mathrm{ab}$ & 97 \\
\hline $\mathrm{FMC} \mathrm{SH}+\mathrm{TCH}$ & $12,8 \mathrm{a}$ & 104 & $20,3 \mathrm{ab}$ & 88 & $17,1 \mathrm{ab}$ & 104 & $23,3 \mathrm{a}$ & 104 & $60,7 \mathrm{ab}$ & 98 \\
\hline $\mathrm{ASH}$ & $5,3 \mathrm{~b}$ & 43 & $11,4 \mathrm{~b}$ & 50 & $13,5 \mathrm{~b}$ & 82 & 25,6 a & 114 & $50,5 \mathrm{~b}$ & 82 \\
\hline $\mathrm{ACH}$ & $5,8 \mathrm{~b}$ & 48 & $16,4 \mathrm{ab}$ & 71 & $21,9 \mathrm{a}$ & 133 & $26,4 \mathrm{a}$ & 117 & $64,7 \mathrm{a}$ & 104 \\
\hline $\mathrm{ASH}+\mathrm{TSH}$ & $4,9 \mathrm{~b}$ & 40 & $11,3 \mathrm{~b}$ & 49 & $19,4 \mathrm{ab}$ & 118 & $22,9 \mathrm{a}$ & 102 & $53,5 \mathrm{ab}$ & 86 \\
\hline $\mathrm{ASH}+\mathrm{TCH}$ & $4,8 \mathrm{~b}$ & 39 & $18,8 \mathrm{ab}$ & 82 & $19,5 \mathrm{ab}$ & 118 & $23,7 \mathrm{a}$ & 105 & $62,1 \mathrm{ab}$ & 100 \\
\hline $\mathrm{ACH}+\mathrm{TSH}$ & $6,6 \mathrm{~b}$ & 54 & $25,4 \mathrm{a}$ & 110 & $17,8 \mathrm{ab}$ & 108 & $22,0 \mathrm{a}$ & 98 & $65,2 \mathrm{a}$ & 105 \\
\hline $\mathrm{ACH}+\mathrm{TCH}$ & $6,5 \mathrm{~b}$ & 53 & $21,1 \mathrm{ab}$ & 92 & $18,0 \mathrm{ab}$ & 109 & $22,1 \mathrm{a}$ & 98 & $61,3 \mathrm{ab}$ & 99 \\
\hline ATCH & $6,4 \mathrm{~b}$ & 52 & $18,6 \mathrm{ab}$ & 81 & $18,8 \mathrm{ab}$ & 114 & 25,9 a & 115 & $63,4 \mathrm{ab}$ & 102 \\
\hline $\mathrm{TSH}$ & $1,3 \mathrm{c}$ & 10 & $0,3 \mathrm{c}$ & 1 & - & - & - & - & $0,3 \mathrm{c}$ & 1 \\
\hline $\mathrm{TCH}$ & $1,5 \mathrm{c}$ & 12 & $0,3 \mathrm{c}$ & 1 & - & - & - & & $0,3 \mathrm{c}$ & 1 \\
\hline TapSH & $1,3 \mathrm{c}$ & 11 & $0,3 \mathrm{c}$ & 1 & - & - & - & - & $0,3 \mathrm{c}$ & 1 \\
\hline TapCH & $1,8 \mathrm{c}$ & 15 & $0,5 \mathrm{c}$ & 2 & - & - & - & - & $0,6 \mathrm{c}$ & 1 \\
\hline TapSH + TSH & $1,5 \mathrm{c}$ & 12 & $0,5 \mathrm{c}$ & 2 & - & - & - & - & $0,5 \mathrm{c}$ & 1 \\
\hline $\mathrm{TapSH}+\mathrm{TCH}$ & $1,4 \mathrm{c}$ & 11 & $0,4 \mathrm{c}$ & 2 & - & - & - & - & $0,4 \mathrm{c}$ & 1 \\
\hline TapCH + TSH & $1,7 \mathrm{c}$ & 14 & $0,5 \mathrm{c}$ & 2 & - & - & - & - & $0,5 \mathrm{c}$ & 1 \\
\hline $\mathrm{TapCH}+\mathrm{TCH}$ & $1,7 \mathrm{c}$ & 14 & $0,7 \mathrm{c}$ & 3 & - & - & - & - & $0,7 \mathrm{c}$ & 1 \\
\hline TapTCH & $1,7 \mathrm{c}$ & 14 & $0,6 \mathrm{c}$ & 3 & - & - & - & - & $0,6 \mathrm{c}$ & 1 \\
\hline DMS & 2,01 & & 10,01 & & 7,31 & & 5,81 & & 13,82 & \\
\hline CV (\%) & 14,0 & & 33,7 & & 26,1 & & 15,8 & & 14,7 & \\
\hline
\end{tabular}

Valores seguidos pela mesma letra, na mesma coluna, não diferem estatisticamente entre si pelo teste de Tukey $5 \%$ de probabilidade. 
É reportado que as rochas fosfáticas de elevada reatividade, geralmente de origem sedimentar, contém em sua estrutura muitas substituições de carbonatos por fosfatos, o que leva à maior solubilidade e, consequentemente, maior eficiência agronômica em determinadas condições de solo, clima, cultura e manejo. As jazidas fosfáticas nacionais são constituídas principalmente de materiais de origem ígnea e de baixa solubilidade e, consequentemente, com baixa eficiência agronômica quando aplicadas in natura (AlCARDE e PONCHIO, 1980; GoEdert e Lobato, 1980).

Não houve diferença significativa quando foi aplicada a rocha fosfática de Arad submetida e não submetida ao processo, visto que esse não foi eficiente em aumentar a solubilidade do material, refletindo em produção semelhante no primeiro cultivo.

A aplicação do rejeito de Tapira, independentemente da exposição ao equipamento ou da adição de material orgânico, proporcionou produções muito baixas das plantas de milho, similares ao controle. Esse fato é decorrente de nesse material haver teor de $\mathrm{P}$ solúvel muito baixo, insuficiente ao desenvolvimento das plantas. Constatam-se pelos dados que, também para esta fonte, não houve qualquer influência do tratamento Humifert na eficiência agronômica.

A análise dos dados obtidos com as plantas de braquiária, primeiro cultivo (Tabela 6), permite verificar aumento de eficiência do fosfato Arad em relação ao FMC. Enquanto no cultivo de milho houve distinção clara entre as eficiências agronômicas do FMC e fosfato Arad em todas as situações, com maior desempenho proporcionado pelo FMC, no primeiro cultivo de braquiária ocorreu tendência geral de eficiência similar entre as duas fontes.

Em termos de EAR, observa-se em todas as situações com o uso de Arad que o índice foi numericamente maior no primeiro cultivo de braquiária ( $49 \%$ a $110 \%$ ) do que aquele do cultivo de milho (39\% a $54 \%$ ). Numericamente, ainda, observa-se que em algumas situações a eficiência do fosfato de Arad foi similar ao FMC (destacamse $\mathrm{ACH}+\mathrm{TSH}$ e $\mathrm{ACH}+\mathrm{TCH})$. Outra observação interessante no primeiro cultivo de braquiária é a de que o processo Humifert teve efeito para a fonte Arad, com vantagem para aqueles submetidos ao processo. Contrastes ortogonais entre ASH $\times \mathrm{ACH}$ e ASH+TSH $\times \mathrm{ACH}+\mathrm{TCH}$ confirmam isto $(p<0,05)$. Para o material de Tapira não houve qualquer efeito de fonte ou tratamento Humifert, pois as produções de MSPA pelas plantas foram iguais ao do controle sem $P$.
No segundo cultivo de braquiária vários tratamentos não propiciaram o desenvolvimento das plantas, devido à deficiência severa de $\mathrm{P}$ (tratamentos envolvendo aplicação apenas de turfa, de Tap e o controle, sem P). Pela análise estatística dos dados remanescentes (FMC e Arad) verificou-se diferença significativa apenas entre $\mathrm{ACH}$ versus $\mathrm{ASH}$, com vantagem para $\mathrm{ACH}$, confirmando assim a eficácia do processo Humifert para o fosfato de Arad. A presença de turfa junto ao fosfato natural parece ter interagido positivamente na eficiência da mistura, uma vez que mesmo sem passar pelo processo, o fosfato natural neste caso não diferiu do fosfato com Humifert ( $\mathrm{ASH}+\mathrm{TSH}$ ou $\mathrm{ASH}+\mathrm{TCH}$ versus $\mathrm{ACH})$.

O terceiro cultivo de braquiária evidenciou condição similar de MSPA para todas as fontes que mantiveram produção até este cultivo. Ao longo dos cultivos observou-se evolução da eficiência do fosfato Arad, quando comparado ao FMC. Em alguns casos houve, numericamente, tendência de Arad > FMC, o que, entretanto, não se confirmou em termos estatísticos. Pela soma dos três cultivos de braquiária revela-se que as fontes FMC e Arad foram superiores aos demais tratamentos (Turfa, Tap e controle), sem haver, no geral, diferenças significativas entre FMC e Arad. As únicas diferenças existentes entre os tratamentos, contendo estas duas fontes, evidenciaram que o tratamento do Arad com Humifert elevou a eficiência desta fonte em relação à mesma fonte sem Humifert (ACH ou ACH+TSH > ASH).

\section{CONCLUSÃO}

Houve aumento de eficiência agronômica das misturas de Patos de Minas e Arad com turfa submetidas ao processo Humifert, em relação à aplicação dos materiais não submetidos ao processo, entretanto, o aumento de solubilidade das fontes tratadas pelo processo foi de pequena magnitude. Apesar dos dados de efeito residual para braquiária terem indicado efeito positivo do processo para o fosfato de Arad, os resultados ainda não podem confirmar a expectativa de utilização desta técnica para produção de fontes alternativas de fósforo de forma econômica, sendo necessários mais estudos, envolvendo modificações na produção dos compostos Humifert.

\section{AGRADECIMENTOS}

Os autores agradecem ao Dr. Jean-Louis Lacout (Institut National Polytechnique de Toulouse, França), que forneceu todas as indicações técnicas necessárias à montagem do equipamento Humifert, à 
FAPESP pelo auxílio à Pesquisa que possibilitou montar o equipamento Humifert no Brasil (processo 03/06545-7), à CAPES pelo apoio financeiro no Doutoramento da primeira autora e ao $\mathrm{CNPq}$ pela concessão de bolsa de produtividade científica à quarta autora do artigo e bolsa de Doutoramento à terceira autora.

\section{REFERÊNCIAS}

ALCARDE, J.C.; PONCHIO, C.O. Caracterização das solubilidades das rochas fosfatadas brasileiras e termofosfatos em diferentes extratores químicos. Revista Brasileira de Ciência do Solo, v.4, p.196-200, 1980.

BIDEGAIN, R. Composition d'un amendement organophosphate obtenu par traitement aux oxides d'azote de composes lingo-cellulosiques. Valeur agronomique comparée à un compost. 1995. 201p. Thèse (Doctorat en Sciences des Agroressources) - Institut National Polytechnique de Toulouse, Toulouse.

BRASIL. Ministério da Agricultura. Análise de corretivos, fertilizantes e inoculantes: métodos oficiais. Brasília: Secretaria Nacional de Defesa Agropecuária, 2004. 104p.

EMBRAPA. Manual de métodos de análise de solo. 2.ed. Rio de Janeiro, 1997. 212p.

EMBRAPA. Sistema Brasileiro de Classificação de Solos. Brasília, 1999. 412p.

GOEDERT, W.J.; LOBATO, E. Eficiência agronômica de fosfatos em solo de cerrado. Pesquisa Agropecuária Brasileira, v.15, p.311-318, 1980.

HOROWITZ, N.; MEURER, E.J. Eficiência de dois fosfatos naturais farelados em função do tamanho de partícula. Ciência Rural, v.33, p.41-47, 2003.

NARDI, S.; TOSONI, M.; PIZZEGHELLO, D.; PROVENZANO, M.R.; CILENTI, A.; STRURARO, A.; RELLA, R.; VIANELLO, A. Chemical characterisitics and biological activity of organic substances extracted from soils by root exsudates. Soil Science Society of America Journal, v.29, p.2012-2019, 2005.

OBA, C.A.I. Aproveitamento de materiais fosfáticos marginais para a produção de fertilizantes organo-fosfatados. 2000.175p. Tese (Doutorado em Engenharia Mineral) - Escola Politécnica, Universidade de São Paulo, São Paulo.

PROCHNOW, L.I.; CHIEN, S.H.; CARMONA, G.; HENAO J. Greenhouse evaluation of phosphorus sources produced from a low-reactive brazilian phosphate rock. Agronomy Journal, v.95, p.293-302, 2003.

RAIJ, B.van; QUAGGIO, J.A. Métodos de análise de solo para fins de fertilidade. Campinas: Instituto Agronômico, 1983. 31p.

RAIJ, B. van; ANDRADE, J.C.; CANTARELLA, H.; QUAGGIO, J.A. Análise química para avaliação da fertilidade de solos tropicais. Campinas: Instituto Agronômico, 2001. 285p.
ROUQUET, N. Role des composes humiques sur la dissolution et la cristalisation du phosphate dicalcique dihydrate (Brushite). 1989. 145p. Thèse (Doctorat en Sciences des Agroressources) - Institut National Polytechnique de Toulouse, Toulouse.

SILVEROL, A.C. Processo Humifert para fertilizantes alternativos organo-fosfatados: obtenção a partir do minério de Angico dos Dias, caracterização dos compostos e avaliação da eficiência agronômica. 2006. 179p. Dissertação (Mestrado em Geoquímica e Geotectônica) - Universidade São Paulo, São Paulo.

SILVEROL, A.C.; TOLEDO, M.C.M.; BENEDITO, D.S.; PROCHNOW, L.I. Compostos organofosfatados obtidos pelo processo Humifert. Geochemica Brasiliensis, v.21, p.8698, 2007.

STERNICHA, F. Matière organique accroissant la proportion de phosphore assimilable dans lês engrais chimiques classiques et dans les phosphates naturels. Brevet $n^{\circ} 87,13177$, França. 1988.

TOLEDO, M. C. M. de, SILVEROL, A. C., BENEDITO, D. S. \& PROCHNOW, L. I. Developpement du procédé Humifert pour obtention des composés organo phosphatés au Brésil. In: INTERNATIONAL CONFERENCE ON THE VALORIZATION OF PHOSPHATES AND PHOSPHOROUSCOMPOUNDS, 2006, Marrakech. Proceedings... Marrakech: Réseau des Chercheurs sur les phosphates, 2006. 